\title{
Matrix Fourier Transforms for Consistent Mathematical Models
}

\author{
Oleg Yaremko and Natalia Yaremko \\ Penza State University, Krasnaya Street 40, 440038 Penza, Russia \\ Correspondence should be addressed to Natalia Yaremko; yaremki@yandex.ru
}

Received 29 April 2016; Accepted 18 July 2016

Academic Editor: Xiao-wei Gao

Copyright (C) 2016 O. Yaremko and N. Yaremko. This is an open access article distributed under the Creative Commons Attribution License, which permits unrestricted use, distribution, and reproduction in any medium, provided the original work is properly cited.

We create a matrix integral transforms method; it allows us to describe analytically the consistent mathematical models. An explicit constructions for direct and inverse Fourier matrix transforms with discontinuous coefficients are established. We introduce special types of Fourier matrix transforms: matrix cosine transforms, matrix sine transforms, and matrix transforms with piecewise trigonometric kernels. The integral transforms of such kinds are used for problems solving of mathematical physics in homogeneous and piecewise homogeneous media. Analytical solution of iterated heat conduction equation is obtained. Stress produced in the elastic semi-infinite solid by pressure is obtained in the integral form.

\section{Introduction}

Matrix integral Fourier transforms with sine, cosine, and piecewise trigonometric kernels represent an important branch of mathematical analysis. It is based on the expansion of a function over a set of cosine or sine basis functions. Integral Fourier transforms of such kinds have shown their special applicability in description of consistent mathematical models. To show the versatility of these transforms, we solve the problems of mathematical physics in homogeneous and piecewise homogeneous media. We find analytical solutions of iterated heat conduction equation and solve a problem about stress in the elastic semi-infinite solid.

Given a real function $f(x)$, which is defined over the positive real line $x \geq 0$, for $\lambda \geq 0$, which is piecewise continuous and absolutely integrable over $[0, \infty)$, the Fourier cosine transform of $f(x)$ is defined as

$$
F[f](\lambda)=\tilde{f}(\lambda)=\int_{0}^{\infty} \cos \lambda x f(x) d x
$$

subject to the existence of the integral. The inverse Fourier cosine transform is given by

$$
F^{-1}[\tilde{f}](\lambda)=f(x)=\frac{2}{\pi} \int_{0}^{\infty} \cos \lambda x \tilde{f}(\lambda) d \lambda
$$

again subject to the existence of the integral used in the definition. The functions $f(x)$ and $\tilde{f}(\lambda)$, if they exist, are said to form a Fourier cosine transform pair.

Given a real function $f(x)$, which is defined over the positive real line $x \geq 0$, for $x \geq 0$, which is piecewise continuous and absolutely integrable over $[0, \infty)$, the Fourier sine transform of $f(x)$ is defined as

$$
F[f](\lambda)=\tilde{f}(\lambda)=\int_{0}^{\infty} \sin \lambda x f(x) d x
$$

subject to the existence of the integral. The inverse Fourier transform is given by

$$
F^{-1}[\tilde{f}](\lambda)=f(x)=\frac{2}{\pi} \int_{0}^{\infty} \sin \lambda x \tilde{f}(\lambda) d \lambda
$$

again subject to the existence of the integral used in the definition. The functions $f(x)$ and $\tilde{f}(\lambda)$, if they exist, are said to form a Fourier transform pair.

Given a real function $f(x)$, which is defined over the positive real line $x \geq 0$, for $x \geq 0$, which is piecewise continuous and absolutely integrable over $[0, \infty)$, the Fourier type transform of $f(x)$ is defined as

$$
F[f](\lambda)=\tilde{f}(\lambda)=\int_{0}^{\infty}(h \sin \lambda x-\lambda \cos \lambda x) f(x) d x
$$

subject to the existence of the integral. 
Theorem 1. The unit normalization constant used here provides a definition for the inverse Fourier type transform, given by

$$
F^{-1}[\tilde{f}](\lambda)=\frac{2}{\pi} \int_{0}^{\infty} \tilde{f}(\lambda) \frac{h \sin \lambda x-\lambda \cos \lambda x}{h^{2}+\lambda^{2}} d \lambda
$$

again subject to the existence of the integral used in the definition.

Proof. Let function $f(x)$ take the following form:

$$
f(x)=\frac{2}{\pi} \int_{0}^{\infty} \tilde{f}(\lambda) \frac{h \sin \lambda x-\lambda \cos \lambda x}{h^{2}+\lambda^{2}} d \lambda .
$$

Then,

$$
h f(x)+f^{\prime}(x)=\frac{2}{\pi} \int_{0}^{\infty} \tilde{f}(\lambda) \sin \lambda x d \lambda .
$$

Due to the inverse Fourier cosine transform, we get

$$
\begin{aligned}
\tilde{f}(\lambda)= & \int_{0}^{\infty} \sin \lambda x\left(h f(x)+f^{\prime}(x)\right) d \lambda \\
= & \left.\sin \lambda x f(x)\right|_{0} ^{\infty} \\
& +\int_{0}^{\infty}(h \sin \lambda x-\lambda \cos \lambda x) f(x) d x \\
= & \int_{0}^{\infty}(h \sin \lambda x-\lambda \cos \lambda x) f(x) d x .
\end{aligned}
$$

The general theory of linear integral transform with some of its applications gave an account of [1-12].

In order to define integral Fourier matrix transforms with piecewise trigonometric kernels, we consider SturmLiouville matrix problem:

$$
-\frac{d}{d x}\left(A(x) \frac{d}{d x} \varphi(x, \lambda)\right)=\lambda^{2} \varphi(x, \lambda), \quad x \in I_{n}^{+},
$$

where

$$
\varphi(x, \lambda)=\sum_{j=1}^{n+1}\left(\theta\left(x-l_{j-1}\right)-\theta\left(x-l_{j}\right)\right) \varphi_{j}(x, \lambda),
$$

bounded nontrivial unknown matrix function of size $r \times$ $r$ called matrix eigenfunction of Sturm-Liouville problem, $A(x)$ is the matrix-valued function of size $r \times r$, and

$$
I_{n}^{+}=\left\{x: x \in \bigcup_{j=1}^{n+1}\left(l_{j-1}, l_{j}\right), l_{0}=0, l_{n+1}=\infty\right\} .
$$

In general, Sturm-Liouville matrix problem does not possess an analytical solution. Therefore, we consider the SturmLiouville piecewise approximation as follows.

$A(x)$ is piecewise constant; that is,

$$
A(x)=\sum_{j=1}^{n+1} A_{i}^{2}\left(\theta\left(x-l_{j-1}\right)-\theta\left(x-l_{j}\right)\right),
$$

where $x=l_{j}, j=1, \ldots, n+1$, are points of discontinuity in $I_{n}^{+}$, and $\theta(x)$ is the Heaviside step function.

The elements of matrix eigenfunctions $\varphi(x, \lambda)$ of a Sturm-Liouville matrix problem are piecewise trigonometric functions. The explicit expression of spectral matrix-valued function $\varphi(x, \lambda)$ allows for defining direct integral Fourier matrix transform with piecewise trigonometric kernels. The explicit solution of dual Sturm-Liouville matrix problem serves as a kernel for an inverse integral Fourier matrix transform.

Integral transforms arise in a natural way through the principle of linear superposition in constructing integral representations of linear differential equations solutions [1315]. The theory of integral Fourier transforms with piecewise trigonometric kernels in a scalar case was studied by Ufljand [16], Najda [17], Procenko and Solov'jov [18], and Lenjuk [19]. The matrix version is adapted for the problems solving in piecewise homogeneous medium and has been developed by Yaremko in $[10,20]$. The necessary proofs by method of contour integration were conducted in [11,21]. It is clear that this method is effective to obtain the exact solution of boundary value problems for piecewise homogeneous media.

\section{Matrix Fourier Transforms with Piecewise Trigonometric Kernels}

The Sturm-Liouville matrix problem [1] is to find the nontrivial solution $y$ bounded on the set $I_{n}^{+}$

$$
y(x, \lambda)=\sum_{k=1}^{n+1} \theta\left(x-l_{k-1}\right) \theta\left(l_{k}-x\right) y_{k}(x, \lambda),
$$

to a system of an ordinary differential equations with constant matrix coefficients

$$
\left(A_{m}^{2} \frac{d^{2}}{d x^{2}}+\lambda^{2} E\right) y_{m}=0, \quad m=\overline{1, n+1}
$$

with the boundary conditions at the points $l_{0}$ and $l_{n+1}$,

$$
\begin{array}{r}
\left(\left(\alpha_{11}^{0}+\lambda^{2} \delta_{11}^{0}\right) \frac{d}{d x}+\left(\beta_{11}^{0}+\lambda^{2} \gamma_{11}^{0}\right)\right) y_{1}=0, \\
\left.\left\|y_{n+1}\right\|\right|_{x=\infty}<\infty,
\end{array}
$$

and internal boundary conditions at the points $x=l_{k}$

$$
\begin{aligned}
& \left(\left(\alpha_{j 1}^{k}+\lambda^{2} \delta_{j 1}^{k}\right) \frac{d}{d x}+\left(\beta_{j 1}^{k}+\lambda^{2} \gamma_{j 1}^{k}\right)\right) y_{k} \\
& \quad=\left(\left(\alpha_{j 2}^{k}+\lambda^{2} \delta_{j 2}^{k}\right) \frac{d}{d x}+\left(\beta_{j 2}^{k}+\lambda^{2} \gamma_{j 2}^{k}\right)\right) y_{k+1},
\end{aligned}
$$

where

$$
\alpha_{j i}^{k}, \beta_{j i}^{k}, \gamma_{j i}^{k}, \delta_{j i}^{k}, A_{j}
$$


are matrices of size $r \times r$ :

$$
\begin{aligned}
y_{m}(x, \lambda) & =\left(\begin{array}{c}
y_{1 m}(x, \lambda) \\
\vdots \\
y_{r m}(x, \lambda)
\end{array}\right), \\
\left\|y_{m}\right\| & =\sqrt{y_{1 m}^{2}+\cdots+y_{r m}^{2}} .
\end{aligned}
$$

Let $y$ be nontrivial solution to boundary value problems (52)(69) for some $\lambda$. The number $\lambda$ is called an eigenvalue, and the corresponding solution $y(x, \lambda)$ is called matrix-valued eigenfunction.

We will required invertible conditions

$$
\operatorname{det} M_{m k} \neq 0, \quad \lambda \in[0, \infty)
$$

for matrices

$$
M_{m k} \equiv\left(\begin{array}{ll}
\beta_{1 m}^{k}+\lambda^{2} \gamma_{1 m}^{k} & \alpha_{1 m}^{k}+\lambda^{2} \delta_{1 m}^{k} \\
\beta_{2 m}^{k}+\lambda^{2} \gamma_{2 m}^{k} & \alpha_{2 m}^{k}+\lambda^{2} \delta_{2 m}^{k}
\end{array}\right) .
$$

The matrices $A_{m}^{2}$ are positive-defined [4]. We denote

$$
\begin{gathered}
\Phi_{n+1}(x)=e^{q_{n+1} x i} \\
\Psi_{n+1}(x)=e^{-q_{n+1} x i} \\
q_{n+1}^{2}=\lambda^{2} A_{n+1}^{-2} .
\end{gathered}
$$

We define the other $n$ pairs of matrix-value functions $\left(\Phi_{k}, \Psi_{k}\right), k=\overline{1, n}$ by the induction relations:

$$
\begin{aligned}
& {\left[\left(\alpha_{j 1}^{k}+\lambda^{2} \delta_{j 1}^{k}\right) \frac{d}{d x}+\left(\beta_{j 1}^{k}+\lambda^{2} \gamma_{j 1}^{k}\right)\right]\left(\Phi_{k} \Psi_{k}\right)} \\
& =\left[\left(\alpha_{j 2}^{k}+\lambda^{2} \delta_{j 2}^{k}\right) \frac{d}{d x}+\left(\beta_{j 2}^{k}+\lambda^{2} \gamma_{j 2}^{k}\right)\right]\left(\Phi_{k+1} \Psi_{k+1}\right) .
\end{aligned}
$$

Introduce the following notations:

$$
\begin{aligned}
\Phi_{0,1}(\lambda)= & \left(\alpha_{11}^{0}+\lambda^{2} \delta_{11}^{0}\right) \Phi_{1}^{\prime}\left(l_{0}, \lambda\right) \\
& +\left(\beta_{11}^{0}+\lambda^{2} \gamma_{11}^{0}\right) \Phi_{1}\left(l_{0}, \lambda\right), \\
\Psi_{0,1}(\lambda)= & \left(\alpha_{11}^{0}+\lambda^{2} \delta_{11}^{0}\right) \Psi_{1}^{\prime}\left(l_{0}, \lambda\right) \\
& +\left(\beta_{11}^{0}+\lambda^{2} \gamma_{11}^{0}\right) \Psi_{1}\left(l_{0}, \lambda\right), \\
\Omega_{k}= & \left(\begin{array}{cc}
\Phi_{k} & \Psi_{k} \\
\Phi_{k}^{/} & \Psi_{k}^{\prime}
\end{array}\right) .
\end{aligned}
$$

Lemma 2. The following identity

$$
\begin{aligned}
& \left(\Omega_{k}^{-1}(x, \lambda)\right)^{\prime}=\left(\begin{array}{cc}
0 & E \\
-q_{k}^{2} & 0
\end{array}\right) \Omega_{k}, \\
& \left(\Omega_{k}^{-1}(x, \lambda)\right)^{\prime \prime}=-\Omega_{k}^{-1} \lambda^{2} A_{k}^{-2}
\end{aligned}
$$

holds true for $k=1, \ldots, n$.
Proof. Using formula [20]

$$
\left(\Omega_{k}^{-1}\right)^{\prime}=-\Omega_{k}^{-1} \Omega_{k}^{\prime} \Omega_{k}^{-1}
$$

and identity

$$
\left(\Omega_{k}\right)^{\prime}=\left(\begin{array}{cc}
\Phi_{k}^{\prime} & \Psi_{k}^{\prime} \\
\Phi_{k}^{\prime \prime} & \Psi_{k}^{\prime \prime}
\end{array}\right)=\left(\begin{array}{cc}
0 & E \\
-q_{k}^{2} & 0
\end{array}\right) \Omega_{k}
$$

we can conclude

$$
\begin{aligned}
\left(\Omega_{k}^{-1}\right)^{\prime} & =-\Omega_{k}^{-1}\left(\begin{array}{cc}
0 & \mathrm{E} \\
-q_{k}^{2} & 0
\end{array}\right) \\
\left(\Omega_{k}^{-1}\right)^{\prime \prime} & =\Omega_{k}^{-1}\left(\begin{array}{cc}
0 & \mathrm{E} \\
-q_{k}^{2} & 0
\end{array}\right)\left(\begin{array}{cc}
0 & \mathrm{E} \\
-q_{k}^{2} & 0
\end{array}\right) \\
& =\Omega_{k}^{-1}\left(\begin{array}{cc}
-q_{k}^{2} & 0 \\
0 & -q_{k}^{2}
\end{array}\right) .
\end{aligned}
$$

Lemma 3. If the following inequality

$$
\operatorname{det}\left(\begin{array}{cc}
\beta_{11}^{0}+\lambda^{2} \gamma_{11}^{0} & \alpha_{11}^{0}+\lambda^{2} \delta_{11}^{0} \\
0 & E
\end{array}\right) \neq 0, \quad \lambda \in[0, \infty)
$$

holds true, then

$$
\begin{aligned}
& \operatorname{det} \Phi_{0,1}(\lambda) \neq 0, \\
& \operatorname{det} \Psi_{0,1}(\lambda) \neq 0,
\end{aligned}
$$

$$
\lambda \in[0, \infty) .
$$

Proof. Matrices $\Phi_{0,1}$ and $\Psi_{0,1}$ are nonsingular due to the following identity:

$$
\begin{aligned}
& \left(\begin{array}{cc}
\Phi_{0,1} & \Psi_{0,1} \\
\Phi_{1}^{\prime} & \Psi_{1}^{\prime}
\end{array}\right) \\
& \quad=\left(\begin{array}{cc}
\beta_{11}^{0}+\lambda^{2} \gamma_{11}^{0} & \alpha_{11}^{0}+\lambda^{2} \delta_{11}^{0} \\
0 & E
\end{array}\right)\left(\begin{array}{ll}
\Phi_{1} & \Psi_{1} \\
\Phi_{1}^{\prime} & \Psi_{1}^{\prime}
\end{array}\right) .
\end{aligned}
$$

Theorem 4. The spectrum of problems (15), (16), and (18) is continuous and fills semiaxis $(0, \infty)$. Sturm-Liouville problem is $r$ time singular. Exactly $r$ linearly independent matrix-valued functions correspond to each eigenvalue $\lambda$. It is possible to take $r$ columns of matrix-value functions:

$$
\begin{aligned}
v(x, \lambda) & =\sum_{k=1}^{n+1} \theta\left(x-l_{k-1}\right) \theta\left(l_{k}-x\right) v_{k}(x, \lambda), \\
v_{j}(x, \lambda) & =\Phi_{j}(x, \lambda) \Phi_{0,1}^{-1}(\lambda)-\Psi_{j}(x, \lambda) \Psi_{0,1}^{-1}(\lambda) .
\end{aligned}
$$

That is

$$
y_{m}(x, \lambda)=\left(\begin{array}{c}
v_{1 m}(x, \lambda) \\
\vdots \\
v_{r m}(x, \lambda)
\end{array}\right) .
$$

Theorem 4 follows from Lemmas 2 and 3. 
Now we consider the dual matrix Sturm-Liouville problem. We find the nontrivial solution $y^{*}$ of a system of ordinary differential equations with constant matrix coefficients

$$
\left(A_{m}^{2} \frac{d^{2}}{d x^{2}}+\lambda^{2} \mathrm{E}\right) y_{m}^{*}=0, \quad m=\overline{1, n+1}
$$

with boundary conditions at the points $x=l_{0}$ and $x=l_{n+1}$

$$
\begin{aligned}
y_{1}^{*}\left(\alpha_{11}^{0}+\lambda^{2} \delta_{11}^{0}\right)^{-1}+\frac{d}{d x} y_{1}^{*}\left(\beta_{11}^{0}+\lambda^{2} \gamma_{11}^{0}\right)^{-1} & =0, \\
\left\|y_{n+1}^{*}\right\| & <\infty,
\end{aligned}
$$

and internal boundary conditions at the points $x=l_{k}$,

$$
\begin{aligned}
& \left(-\left(y_{k}^{*}\right)^{\prime} y_{k}^{*}\right)\left(\begin{array}{ll}
\beta_{11}^{k}+\lambda^{2} \gamma_{11}^{k} & \alpha_{11}^{k}+\lambda^{2} \delta_{11}^{k} \\
\beta_{21}^{k}+\lambda^{2} \gamma_{21}^{k} & \alpha_{21}^{k}+\lambda^{2} \delta_{21}^{k}
\end{array}\right)^{-1} \\
& =\left(-\left(y_{k+1}^{*}\right)^{\prime} y_{k+1}^{*}\right)\left(\begin{array}{ll}
\beta_{12}^{k}+\lambda^{2} \gamma_{12}^{k} & \alpha_{12}^{k}+\lambda^{2} \delta_{12}^{k} \\
\beta_{22}^{k}+\lambda^{2} \gamma_{22}^{k} & \alpha_{22}^{k}+\lambda^{2} \delta_{22}^{k}
\end{array}\right)^{-1}
\end{aligned}
$$

We write the solution of the boundary value problem in the following form:

$$
\begin{aligned}
y^{*}(\xi, \lambda) & =\sum_{k=1}^{n+1} \theta\left(\xi-l_{k-1}\right) \theta\left(l_{k}-\xi\right) y_{k}^{*}(\xi, \lambda), \\
y_{m}^{*}(\xi, \lambda) & =\left(y_{m 1}^{*}(\xi, \lambda) \cdots y_{m r}^{*}(\xi, \lambda)\right), \\
\left\|y_{m}^{*}\right\| & =\sqrt{\left(y_{1 m}^{*}\right)^{2}+\cdots+\left(y_{r m}^{*}\right)^{2}} .
\end{aligned}
$$

Theorem 5. The spectrum of problems (35), (36), and (38) is continuous and fills semiaxis $(0, \infty)$. Sturm-Liouville problem is $r$ time singular. Exactly $r$ linearly independent matrix-valued functions correspond to each eigenvalue $\lambda$. It is possible to take $r$ rows of matrix-value functions:

$$
\begin{aligned}
& v^{*}(x, \lambda)=\sum_{k=1}^{n+1} \theta\left(x-l_{k-1}\right) \theta\left(l_{k}-x\right) v_{k}^{*}(x, \lambda), \\
& v_{j}^{*}(x, \beta)=\left(\Phi_{0,1} \Psi_{0,1}\right) \Omega_{j}^{-1}(x, \beta)\left(\begin{array}{l}
0 \\
E
\end{array}\right) A_{j}^{-2} .
\end{aligned}
$$

That is,

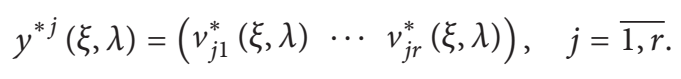

Theorem 5 follows from Lemmas 2 and 3.

The explicit expression of spectral matrix-valued function $u(x, \lambda)$ and the dual spectral function $u^{*}(x, \lambda)$ allow for writing the decomposition theorem on the set $I_{n}^{+}$.

Theorem 6. Let vector-valued function $f(x)$ be defined over $I_{n}^{+}$, continuous, absolutely integrable, and has a bounded variation. Then, for any $x \in I_{n}^{+}$, the decomposition formula

$$
\begin{aligned}
& f(x)=-\frac{1}{\pi i} \int_{0}^{\infty} v(x, \lambda)\left(\int_{l_{0}}^{\infty} v^{*}(\xi, \lambda) f(\xi) d \xi+\gamma_{11}^{0} f_{1}\left(l_{0}\right)+\delta_{11}^{0} f_{1}^{\prime}\left(l_{0}\right)\right. \\
& \left.\quad+\sum_{k=1}^{n}\left(\Phi_{0,1} \Psi_{0,1}\right) \Omega_{k}^{-1}\left(l_{k}, \lambda\right) M_{k 1}^{-1}(\lambda) \cdot\left(\left(\begin{array}{cc}
\gamma_{21}^{k} & \delta_{21}^{k} \\
\gamma_{22}^{k} & \delta_{22}^{k}
\end{array}\right)\left(\begin{array}{c}
f_{k+1}\left(l_{k}\right) \\
f_{k+1}^{\prime}\left(l_{k}\right)
\end{array}\right)-\left(\begin{array}{cc}
\gamma_{11}^{k} & \delta_{11}^{k} \\
\gamma_{12}^{k} & \delta_{12}^{k}
\end{array}\right)\left(\begin{array}{c}
f_{k}\left(l_{k}\right) \\
f_{k}^{\prime}\left(l_{k}\right)
\end{array}\right)\right)\right) \lambda d \lambda
\end{aligned}
$$

holds true.

This theorem can be proved by method of contour integration [17].

We define the direct and inverse matrix integral Fourier transforms on the real semiline with piecewise trigonometric kernels according to Theorem 6.

The direct transform is

$$
\begin{aligned}
F_{n+} & {[f](\lambda)=\int_{l_{0}}^{\infty} v^{*}(\xi, \lambda) f(\xi) d \xi+\gamma_{11}^{0} f_{1}\left(l_{0}\right) } \\
& +\delta_{11}^{0} f_{1}^{\prime}\left(l_{0}\right)+\sum_{k=1}^{n}\left(\Phi_{0,1} \Psi_{0,1}\right) \Omega_{k}^{-1}\left(l_{k}, \lambda\right) M_{k 1}^{-1}(\lambda) \\
& +\left(\left(\begin{array}{cc}
\gamma_{21}^{k} & \delta_{21}^{k} \\
\gamma_{22}^{k} & \delta_{22}^{k}
\end{array}\right)\left(\begin{array}{l}
f_{k+1}\left(l_{k}\right) \\
f_{k+1}^{\prime}\left(l_{k}\right)
\end{array}\right)\right. \\
& \left.-\left(\begin{array}{cc}
\gamma_{11}^{k} & \delta_{11}^{k} \\
\gamma_{12}^{k} & \delta_{12}^{k}
\end{array}\right)\left(\begin{array}{l}
f_{k}\left(l_{k}\right) \\
f_{k}^{\prime}\left(l_{k}\right)
\end{array}\right)\right)
\end{aligned}
$$

and the inverse transform is

$$
F_{n+}^{-1}[\tilde{f}](x)=-\frac{1}{\pi i} \int_{0}^{\infty} \lambda v(x, \lambda) \tilde{f}(\lambda) d \lambda \equiv f(x),
$$

when

$$
f(x)=\sum_{k=1}^{n+1} \theta\left(l_{k}-x\right) \theta\left(x-l_{k-1}\right) f_{k}(x) .
$$

Now we will get the result of the basic identity of matrix integral transforms with piecewise trigonometric kernels for differential operator:

$$
B=\sum_{j=1}^{n+1} \theta\left(x-l_{j-1}\right) \theta\left(l_{j}-x\right) A_{j}^{2} \frac{d^{2}}{d x^{2}} .
$$

Theorem 7. If vector-valued function

$$
f(x)=\sum_{k=1}^{n+1} \theta\left(x-l_{k-1}\right) \theta\left(l_{k}-x\right) f_{k}(x)
$$


is three times continuously differentiable over set $I_{n+}$, has the limit values of its third-order derivatives at the points $x=l_{k}$, and satisfies boundary condition on infinity

$$
\lim _{x \rightarrow \infty}\left(u^{*}(x, \lambda) \frac{d}{d x} f(x)-\frac{d}{d x} u^{*}(x, \lambda) f(x)\right)=0
$$

and internal boundary conditions at the points $x=l_{k}$, then the basic identity

$$
\begin{aligned}
F_{n+} & {[B(f)](\lambda)=-\lambda^{2} \tilde{f}(\lambda)-\left\{\left(\beta_{11}^{0} f_{1}\left(l_{0}\right)\right.\right.} \\
+ & \left.\left.\alpha_{11}^{0} f_{1}^{\prime}\left(l_{0}\right)\right)-\left(\gamma_{11}^{0} A_{1}^{2} f_{1}^{\prime \prime}\left(l_{0}\right)+\delta_{11}^{0} A_{1}^{2} f_{1}^{\prime \prime \prime}\left(l_{0}\right)\right)\right\} \\
& -\sum_{k=1}^{n}\left(\Phi_{0,1} \Psi_{0,1}\right) \Omega_{k}^{-1}\left(l_{k}, \lambda\right) M_{k 1}^{-1}(\lambda) \\
& .\left\{\left(\left(\begin{array}{ll}
\beta_{21}^{k} & \alpha_{21}^{k} \\
\beta_{22}^{k} & \alpha_{22}^{k}
\end{array}\right)\left(\begin{array}{c}
f_{k+1}\left(l_{k}\right) \\
f_{k+1}^{\prime}\left(l_{k}\right)
\end{array}\right)\right.\right. \\
& \left.-\left(\begin{array}{ll}
\gamma_{21}^{k} & \delta_{21}^{k} \\
\gamma_{22}^{k} & \delta_{22}^{k}
\end{array}\right)\left(\begin{array}{l}
A_{k+1}^{2} f_{k+1}^{\prime \prime}\left(l_{k}\right) \\
A_{k+1}^{2} f_{k+1}^{\prime \prime \prime}\left(l_{k}\right)
\end{array}\right)\right) \\
& -\left(\left(\begin{array}{ll}
\beta_{11}^{k} & \alpha_{11}^{k} \\
\beta_{12}^{k} & \alpha_{12}^{k}
\end{array}\right)\left(\begin{array}{l}
f_{k}\left(l_{k}\right) \\
f_{k}^{\prime}\left(l_{k}\right)
\end{array}\right)\right. \\
& \left.\left.-\left(\begin{array}{ll}
\gamma_{11}^{k} & \delta_{11}^{k} \\
\gamma_{12}^{k} & \delta_{12}^{k}
\end{array}\right)\left(\begin{array}{l}
A_{k}^{2} f_{k}^{\prime}\left(l_{k}\right) \\
A_{k}^{2} f_{k}^{\prime \prime \prime}\left(l_{k}\right)
\end{array}\right)\right)\right\}
\end{aligned}
$$

holds true.

This theorem can be proved by method of integration by parts.

\section{Special Types of Matrix Integral Fourier Transforms}

Theorem 8. The matrix-valued Sturm-Liouville problem with Dirichlet boundary condition

$$
\begin{aligned}
-A^{2} \frac{d^{2}}{d x^{2}} \varphi(x, \lambda) & =\lambda^{2} \varphi(x, \lambda), \quad 0<x<\infty, \\
\varphi(0, \lambda) & =0, \\
\|\varphi(x, \lambda)\| & <\infty
\end{aligned}
$$

provides the direct and inverse matrix integral sine transforms on the real semiline:

$$
\begin{gathered}
F_{+}[f](\lambda) \equiv \tilde{f}(\lambda)=\int_{0}^{\infty} \sin \left(A^{-1} \lambda \xi\right) f(\xi) d \xi \\
f(x)=\frac{2 A^{-1}}{\pi} \int_{0}^{\infty} \sin \left(A^{-1} \lambda x\right) \tilde{f}(\lambda) d \lambda
\end{gathered}
$$

Proof. Performing calculations in formulas (33) and (41), we get

$$
\begin{aligned}
& \Phi(x, \lambda)=\exp \left(i A^{-1} \lambda x\right) \\
& \Psi(x, \lambda)=\exp \left(-i A^{-1} \lambda x\right), \\
& \Phi_{0,1}=\mathrm{E}, \\
& \Psi_{0,1}=\mathrm{E} \text {, } \\
& v(x, \lambda)=\Phi(x, \lambda)-\Psi(x, \lambda)=2 i \sin \left(A^{-1} \lambda x\right), \\
& v^{*}(x, \beta)=(\mathrm{EE})\left(\begin{array}{cc}
\Phi & \Psi \\
\Phi^{\prime} & \Psi^{\prime}
\end{array}\right)^{-1}\left(\begin{array}{l}
0 \\
\mathrm{E}
\end{array}\right) A^{-2} \\
& =-\frac{A}{2 i}(\mathrm{EE})\left(\begin{array}{cc}
-i A^{-1} \lambda x e^{-i A^{-1} \lambda x} & -e^{-i A^{-1} \lambda x} \\
-i A^{-1} \lambda x e^{i A^{-1} \lambda x} & e^{i A^{-1} \lambda x}
\end{array}\right) \\
& \cdot\left(\begin{array}{l}
0 \\
\mathrm{E}
\end{array}\right) A^{-2}=A^{-1} \sin \left(A^{-1} \lambda x\right) \text {. }
\end{aligned}
$$

Theorem 9. The matrix-valued Sturm-Liouville problem with Neumann boundary condition

$$
\begin{aligned}
-A^{2} \frac{d^{2}}{d x^{2}} \varphi(x, \lambda) & =\lambda^{2} \varphi(x, \lambda), \quad 0<x<\infty, \\
\varphi^{\prime}(0, \lambda) & =0, \\
\|\varphi(x, \lambda)\| & <\infty
\end{aligned}
$$

provides the direct and inverse matrix integral cosine transforms on the real semiline:

$$
\begin{array}{r}
F_{+}[f](\lambda) \equiv \widetilde{f}(\lambda)=\int_{0}^{\infty} \cos \left(A^{-1} \lambda \xi\right) f(\xi) d \xi, \\
f(x)=\frac{2 A^{-1}}{\pi} \int_{0}^{\infty} \cos \left(A^{-1} \lambda x\right) \tilde{f}(\lambda) d \lambda .
\end{array}
$$

Theorem 10. The matrix-valued Sturm-Liouville problem with Robin boundary condition

$$
\begin{aligned}
& -A^{2} \frac{d^{2} \varphi}{d x^{2}}=\lambda^{2} \varphi(x, \lambda), \quad x>0, \\
& H \varphi+\frac{d \varphi}{d x}=0, \quad x=0, \\
& \|\varphi(x, \lambda)\|<\infty,
\end{aligned}
$$


where $H$ is the square matrix with negative eigenvalues, provides the direct and inverse matrix Fourier type transforms on the real semiline:

$$
\begin{aligned}
& F_{+}[f](\lambda) \equiv \tilde{f}(\lambda) \\
& =\int_{0}^{\infty}\left(H \sin A^{-1} \lambda \xi-A^{-1} \lambda \cos A^{-1} \lambda \xi\right) A f(\xi) d \xi, \\
& f(x) \\
& \quad=\frac{2}{\pi} \int_{0}^{\infty} A\left(H \sin A^{-1} \lambda \xi-A^{-1} \lambda \cos A^{-1} \lambda \xi\right) \\
& \quad \cdot A\left((H A)^{2}+\lambda^{2} \mathrm{E}\right)^{-1} \tilde{f}(\lambda) d \lambda .
\end{aligned}
$$

Proof. Substitute

$$
\begin{aligned}
& \Phi(x, \lambda)=\exp \left(i A^{-1} \lambda x\right), \\
& \Psi(x, \lambda)=\exp \left(-i A^{-1} \lambda x\right)
\end{aligned}
$$

into (33) and (41); then,

$$
\begin{aligned}
& \Phi_{0,1}=H+i \lambda A^{-1} \text {, } \\
& \Psi_{0,1}=H-i \lambda A^{-1} \text {, } \\
& v(x, \lambda) \\
& =\Phi(x, \lambda)\left(H+i \lambda A^{-1}\right)^{-1} \\
& -\Psi(x, \lambda)\left(H-i \lambda A^{-1}\right)^{-1} \\
& =A(\Phi(x, \lambda)(H A-i \lambda \mathrm{E})-\Psi(x, \lambda)(H A+i \lambda \mathrm{E})) \\
& \cdot\left((H A)^{2}+\lambda^{2} \mathrm{E}\right)^{-2} \\
& =2 i A\left(\sin \left(\lambda A^{-1} x\right) H-A^{-1} \lambda \cos \left(A^{-1} \lambda x\right)\right) A \\
& v^{*}(x, \beta)=(\mathrm{EE})\left(\begin{array}{cc}
\Phi & \Psi \\
\Phi^{\prime} & \Psi^{\prime}
\end{array}\right)^{-1}\left(\begin{array}{l}
0 \\
\mathrm{E}
\end{array}\right) A^{-2} \\
& =-\frac{\mathrm{E}}{2 i}\left(H+i \lambda A^{-1} H-i \lambda A^{-1}\right) \\
& \cdot\left(\begin{array}{c}
-e^{-i A^{-1} \lambda x} \\
e^{i A^{-1} \lambda x}
\end{array}\right)\left(\begin{array}{l}
0 \\
\mathrm{E}
\end{array}\right) A^{-1} \\
& =\left(-H \sin \left(\lambda A^{-1} x\right)+A^{-1} \lambda \cos \left(A^{-1} \lambda x\right)\right) A .
\end{aligned}
$$

Theorem 11. The matrix-valued Sturm-Liouville problem with Dirichlet boundary condition on the composite semiline $I_{1+}=$ $(0, l) \cup(l, \infty)$

$$
\begin{aligned}
&-A_{1}^{2} \frac{d^{2} \varphi_{1}}{d x^{2}}=\lambda^{2} \varphi_{1}(x, \lambda), x \in(0, l) ; \\
& A_{2}^{2} \frac{d^{2} \varphi_{2}}{d x^{2}}=\lambda^{2} \varphi_{2}(x, \lambda), \quad x \in(l, \infty) \\
& \varphi_{1}=0, x=0,
\end{aligned}
$$

$$
\begin{aligned}
\varphi_{1} & =\varphi_{2}, \\
\lambda_{1} \partial_{x} \varphi_{1} & =\lambda_{2} \partial_{x} \varphi_{2}, \quad x=l, \\
\left\|\varphi_{2}(x, \lambda)\right\| & <\infty
\end{aligned}
$$

provides the direct and inverse matrix sine type integral transforms on the composite real semiline:

$$
\begin{aligned}
& F_{+}[f](\lambda) \equiv \tilde{f}(\lambda)=\int_{0}^{l} \sin q_{1} \xi \cdot A_{1}^{-1} f_{1}(\xi) d \xi \\
& +\int_{l}^{\infty}\left(\cos q_{1} l \cdot \sin q_{2}(\xi-l)\right. \\
& \left.\quad+\sin q_{1} l \chi \cos q_{2}(\xi-l)\right) A_{2}^{-1} f_{2}(\xi) d \xi, \\
& f_{1}(x)=\frac{2}{\pi} \int_{0}^{\infty} \sin q_{1} x \tilde{f}(\lambda) d \lambda, \\
& f_{2}(x)=\frac{2}{\pi} \int_{0}^{\infty}\left(\cos q_{2}(x-l) \sin q_{1} l\right. \\
& \left.\quad+\sin q_{2}(x-l) \chi^{-1} \cos q_{1} l\right) \Omega \tilde{f}(\lambda) d \lambda,
\end{aligned}
$$

where

$$
\begin{aligned}
& \Omega=\left(\sin q_{1} l \chi \sin q_{1} l+\cos q_{1} l \chi^{-1} \cos q_{1} l\right)^{-1}, \\
& q_{1}=A_{1}^{-1} \lambda_{1} \\
& q_{2}=A_{2}^{-1} \lambda_{1} \cdot \chi=\lambda_{2} A_{2}^{-1} A_{1} \lambda_{1}^{-1} .
\end{aligned}
$$

Proof. Performing calculations in formulas (33) and (41), we get

$$
\begin{aligned}
\Phi_{2} & =\cos q_{2}(x-l)+i \sin q_{2}(x-l), \quad x>l, \\
\Psi_{2} & =\cos q_{2}(x-l)-i \sin q_{2}(x-l), \quad x>l, \\
\Phi_{1} & =\cos q_{1}(x-l)+i \sin q_{1}(x-l) \cdot \chi, \quad 0<x<l, \\
\Psi_{1} & =\cos q_{1}(x-l)-i \sin q_{1}(x-l) \cdot \chi, \quad 0<x<l, \\
\Phi_{0,1} & =\cos q_{1} l-i \sin q_{1} l \cdot \chi, \\
\Psi_{0,1} & =\cos q_{1} l+i \sin q_{1} l \cdot \chi .
\end{aligned}
$$

Then, matrix eigenfunctions of Sturm-Liouville problem have the following form:

$$
\begin{aligned}
& v_{2}=\Phi_{2}(x, \lambda) \Phi_{0,1}^{-1}(\lambda)-\Psi_{2}(x, \lambda) \Psi_{0,1}^{-1}(\lambda) \\
& =2 i\left(\cos q_{2}(x-l) \sin q_{2}(x-l)\right) \\
& \cdot\left(\begin{array}{cc}
\cos q_{1} l & \sin q_{1} l \cdot \chi \\
-\sin q_{1} l \cdot \chi & \cos q_{1} l
\end{array}\right)^{-1}\left(\begin{array}{l}
0 \\
\mathrm{E}
\end{array}\right)=2 i \\
& \cdot\left(\cos q_{2}(x-l) \sin q_{1} l+\sin q_{2}(x-l) \chi^{-1} \cos q_{1} l\right) \\
& \text {. } \Omega
\end{aligned}
$$




$$
\begin{aligned}
& v_{1}=\Phi_{1}(x, \lambda) \Phi_{0,1}^{-1}(\lambda)-\Psi_{1}(x, \lambda) \Psi_{0,1}^{-1}(\lambda) \\
& =2 i\left(\cos q_{1}(x-l) \sin q_{1}(x-l) \chi^{-1}\right) \\
& \cdot\left(\begin{array}{cc}
\cos q_{1} l & \sin q_{1} l \cdot \chi \\
-\sin q_{1} l \cdot \chi & \cos q_{1} l
\end{array}\right)^{-1}\left(\begin{array}{l}
0 \\
\mathrm{E}
\end{array}\right)=2 i \\
& \text { - }\left(\cos q_{2}(x-l) \sin q_{1} l+\sin q_{2}(x-l) \cos q_{1} l\right) \Omega \\
& =2 i \sin q_{1} x \cdot \Omega \text {. }
\end{aligned}
$$

And dual matrix eigenfunctions of Sturm-Liouville problem have the following form:

$$
\begin{aligned}
& v_{1}^{*}(x, \beta)=\left(\Phi_{0,1} \Psi_{0,1}\right) \Omega_{1}^{-1}(x, \beta)\left(\begin{array}{l}
0 \\
\mathrm{E}
\end{array}\right) A_{1}^{-2} \\
& =\left(\Phi_{0,1} \Psi_{0,1}\right) \\
& \cdot\left(\begin{array}{cc}
\cos q_{1}(x-l) & \sin q_{1}(x-l) \chi \\
-q_{1} \sin q_{1}(x-l) \chi & q_{1} \cos q_{1}(x-l)
\end{array}\right)^{-1} \\
& \cdot\left(\begin{array}{l}
0 \\
\mathrm{E}
\end{array}\right) A_{1}^{-2}=\left(\begin{array}{lll}
\cos q_{1} l & \sin q_{1} l
\end{array}\right) \\
& \cdot\left(\begin{array}{cc}
\cos q_{1}(x-l) & \sin q_{1}(x-l) \cdot \chi \\
-q_{1} \sin q_{1}(x-l) \cdot \chi & q_{1} \cos q_{1}(x-l)
\end{array}\right)^{-1} \\
& \text {. }\left(\begin{array}{l}
0 \\
\mathrm{E}
\end{array}\right) A_{1}^{-2}=-\frac{A_{1}^{-1}}{\lambda} \sin q_{1} x, \\
& v_{2}^{*}(x, \beta)=\left(\Phi_{0,1} \Psi_{0,1}\right) \Omega_{2}^{-1}(x, \beta)\left(\begin{array}{l}
0 \\
\mathrm{E}
\end{array}\right) A_{2}^{-2} \\
& =\left(\Phi_{0,1} \Psi_{0,1}\right) \\
& \cdot\left(\begin{array}{cc}
\cos q_{2}(x-l) & \sin q_{2}(x-l) \chi \\
-q_{2} \sin q_{2}(x-l) \chi & q_{2} \cos q_{2}(x-l)
\end{array}\right)^{-1} \\
& \cdot\left(\begin{array}{l}
0 \\
\mathrm{E}
\end{array}\right) A_{2}^{-2}=\left(\begin{array}{lll}
\cos q_{1} l & \sin q_{1} l
\end{array}\right)\left(\begin{array}{cc}
1 & 0 \\
0 & -\chi
\end{array}\right) \\
& \cdot\left(\begin{array}{cc}
\cos q_{2}(x-l) & \sin q_{2}(x-l) \\
-q_{1} \sin q_{2}(x-l) & q_{1} \cos q_{2}(x-l)
\end{array}\right)^{-1} \\
& \cdot\left(\begin{array}{l}
0 \\
\mathrm{E}
\end{array}\right) A_{2}^{-2}=-\left(\cos q_{1} l \sin q_{2}(x-l)+\sin q_{1} l\right. \\
& \text {. } \left.\chi \cos q_{2}(x-l)\right) \frac{A_{2}^{-1}}{\lambda} .
\end{aligned}
$$

Now we can use the matrix sine type integral transforms on the composite real semiline (43) and (44) to describe analytically the consistent mathematical models.

\section{Analytical Solution of Iterated Heat Conduction Equation}

In this section, we can solve a mixed boundary value problem for iterated heat conduction equation [22].

Let

$$
u=\sum_{k=1}^{n+1} \theta\left(x-l_{k-1}\right) \theta\left(l_{k}-x\right) u_{k}(t, x)
$$

be a solution of system of differential equations

$$
\left(\partial_{t}-a_{k}^{2} \partial_{x x}\right)^{2} u_{k}=0
$$

$$
x \in\left(l_{k-1}, l_{k}\right), k=1, \ldots, n+1
$$

with initial conditions

$$
\begin{aligned}
u_{k}(0, x) & =f_{k}(x), \\
\partial_{t} u_{k}(0, x) & =0
\end{aligned}
$$

with boundary conditions

$$
\begin{array}{r}
h_{1} u_{1}+\partial_{x} u_{1}=0, \\
h_{2} \partial_{x x} u_{1}+\partial_{x x x} u_{1}=0,
\end{array}
$$

$$
\text { if } x=0
$$

and internal boundary conditions at the points $x=l_{k}$

$$
\begin{aligned}
u_{k} & =u_{k+1} \\
\lambda_{k} \partial_{x} u_{k} & =\lambda_{k+1} \partial_{x} u_{k+1}, \\
\mu_{k} \partial_{x x} u_{k} & =\mu_{k} \partial_{x x} u_{k+1}, \\
v_{k} \partial_{x x x} u_{k} & =v_{k+1} \partial_{x x x} u_{k+1} .
\end{aligned}
$$

At the beginning, we will solve an auxiliary vector mixed boundary value problem. Let

$$
\left(\begin{array}{l}
u \\
v
\end{array}\right)=\sum_{k=1}^{n+1} \theta\left(x-l_{k-1}\right) \theta\left(l_{k}-x\right)\left(\begin{array}{l}
u_{k} \\
v_{k}
\end{array}\right)
$$

be a solution to the system of the differential equations

$$
\partial_{t}\left(\begin{array}{l}
u_{k} \\
v_{k}
\end{array}\right)=\left(\begin{array}{cc}
a_{k}^{2} & -a_{k}^{2} \\
0 & a_{k}^{2}
\end{array}\right) \partial_{x x}\left(\begin{array}{c}
u_{k} \\
v_{k}
\end{array}\right),
$$

with initial conditions

$$
\left(\begin{array}{l}
u_{k} \\
v_{k}
\end{array}\right)=\left(\begin{array}{l}
f_{k}(x) \\
f_{k}(x)
\end{array}\right), \quad \text { if } t=0
$$

with boundary conditions at the point $x=0$

$$
\left(\begin{array}{cc}
h_{1} & 0 \\
h_{2}-h_{1} & h_{2}
\end{array}\right)\left(\begin{array}{l}
u_{1} \\
v_{1}
\end{array}\right)+\partial_{x}\left(\begin{array}{l}
u_{1} \\
v_{1}
\end{array}\right)=\left(\begin{array}{l}
0 \\
0
\end{array}\right)
$$


and internal boundary conditions at the points $x=l_{k}$

$$
\begin{gathered}
\left(\begin{array}{cc}
1 & 0 \\
\frac{\mu_{k}}{a_{k}^{2}} & \frac{\mu_{k}}{a_{k}^{2}}
\end{array}\right)\left(\begin{array}{l}
u_{k} \\
v_{k}
\end{array}\right)=\left(\begin{array}{cc}
1 & 0 \\
\frac{\mu_{k+1}}{a_{k+1}^{2}} & \frac{\mu_{k+1}}{a_{k+1}^{2}}
\end{array}\right)\left(\begin{array}{c}
u_{k+1} \\
v_{k+1}
\end{array}\right), \\
\left(\begin{array}{cc}
\lambda_{1} & 0 \\
\frac{v_{k}}{a_{k}^{2}} & \frac{v_{k}}{a_{k}^{2}}
\end{array}\right) \partial_{x}\left(\begin{array}{l}
u_{k} \\
v_{k}
\end{array}\right)=\left(\begin{array}{cc}
\lambda_{2} & 0 \\
\frac{v_{k+1}}{a_{k+1}^{2}} & \frac{v_{k+1}}{a_{k+1}^{2}}
\end{array}\right) \partial_{x}\left(\begin{array}{c}
u_{k+1} \\
v_{k+1}
\end{array}\right) .
\end{gathered}
$$

Lemma 12. The solution of problems (72)-(76) has the following form:

$$
\left(\begin{array}{l}
u \\
v
\end{array}\right)=F_{n+}^{-1}\left[e^{-\lambda^{2} t} \tilde{f}(\lambda)\right],
$$

where

$$
\tilde{f}(\lambda)=\int_{0}^{\infty} v^{*}(\xi, \lambda)\left(\begin{array}{l}
f(\xi) \\
f(\xi)
\end{array}\right) d \xi .
$$

Proof. This lemma can be proved by Fourier integral transform method with piecewise trigonometric kernels (43)-(44). Let the vector-function

$$
\left(\begin{array}{c}
\widetilde{u} \\
\widetilde{v}
\end{array}\right) \equiv F_{n+}\left(\begin{array}{l}
u \\
v
\end{array}\right)=\int_{0}^{\infty} v^{*}(\xi, \lambda)\left(\begin{array}{l}
u(\xi) \\
v(\xi)
\end{array}\right) d \xi
$$

be the Fourier transform. Then, from Theorem 7, vectorfunction $\left(\frac{\tilde{u}}{\widetilde{v}}\right)$ will be a solution of the Cauchy problem:

$$
\begin{aligned}
& \frac{d}{d t}\left(\begin{array}{l}
\widetilde{u} \\
\widetilde{v}
\end{array}\right)=-\lambda^{2}\left(\begin{array}{l}
\widetilde{u} \\
\widetilde{v}
\end{array}\right), \\
& \left(\begin{array}{l}
\widetilde{u}(0) \\
\widetilde{v}(0)
\end{array}\right)=F_{n+}\left[\left(\begin{array}{l}
f(x) \\
f(x)
\end{array}\right)\right] .
\end{aligned}
$$

The solution has the following form:

$$
\left(\begin{array}{l}
\widetilde{u} \\
\widetilde{v}
\end{array}\right)=e^{-\lambda^{2} t} F_{n+}\left[\left(\begin{array}{l}
f(x) \\
f(x)
\end{array}\right)\right] .
$$

To complete the proof, we apply inverse Fourier transform $F_{n+}^{-1}$ :

$$
\left(\begin{array}{l}
u \\
v
\end{array}\right)=F_{n+}^{-1}\left[e^{-\lambda^{2} t} \tilde{f}(\lambda)\right] .
$$

Theorem 13. Let $\left(\begin{array}{c}u \\ v\end{array}\right)$ be a solution of vector problems (72)(76); then,

$$
u_{k}=\left(\begin{array}{ll}
1 & 0
\end{array}\right)\left(\begin{array}{l}
u_{k} \\
v_{k}
\end{array}\right), \quad k=1, \ldots, n+1
$$

is a solution to mixed boundary value problems (67)-(70) for iterated heat conduction equation (67).
Proof. In accordance with (72), the function $u$ is a solution of iterated heat conduction equation (67):

$$
\left(\partial_{t}-a^{2} \partial_{x x}\right)^{2} u=a^{2} \partial_{t}\left(\partial_{t}-a^{2} \partial_{x x}\right) v=0 .
$$

Due to (76), we get the initial condition:

$$
\begin{aligned}
\partial_{t} u(0, x) & =a^{2} \partial_{x x} u(0, x)-a^{2} \partial_{x x} v(0, x) \\
& =a^{2} f^{\prime \prime}(x)-a^{2} f^{\prime \prime}(x)=0 .
\end{aligned}
$$

On the basis of (74), the Robin boundary conditions have the following form: the first condition is as follows:

$$
\left(h_{2}-h_{1}\right) u+h_{2} v+\partial_{x} v=0, \quad \text { if } x=0,
$$

and the second condition is as follows: if $x=0$, then

$$
\begin{aligned}
h_{2} \partial_{x x} u+\partial_{x x x} u & =h_{2} \frac{\partial_{t} u+\partial_{t} v}{a^{2}}+\partial_{x} \frac{\partial_{t} u+\partial_{t} v}{a^{2}} \\
& =\frac{h_{2} \partial_{t} u+\partial_{t x} u+\left(h_{2} \partial_{t} v+\partial_{t x} v\right)}{a^{2}} \\
& =\frac{h_{2} \partial_{t} u+\partial_{t x} u+\left(h_{1}-h_{2}\right) \partial_{t} u}{a^{2}} \\
& =\partial_{t} \frac{h_{1} u+\partial_{x} u}{a^{2}}=0 .
\end{aligned}
$$

It follows from (93)-(94) that

$$
\begin{aligned}
\partial_{t} \frac{\mu_{k}\left(u_{k}+v_{k}\right)}{a_{k}^{2}} & =\partial_{t} \frac{\mu_{k+1}\left(u_{k+1}+v_{k+1}\right)}{a_{k+1}^{2}} ; \\
\partial_{x} \partial_{t} \frac{v_{k}\left(u_{k}+v_{k}\right)}{a_{k}^{2}} & =\partial_{x} \partial_{t} \frac{v_{k+1}\left(u_{k+1}+v_{k+1}\right)}{a_{k+1}^{2}} \\
\partial_{t} \frac{u_{i}+v_{i}}{a_{i}^{2}} & =\partial_{x x} u_{i} .
\end{aligned}
$$

Then, the internal boundary conditions at the points $x=l_{k}$

$$
\begin{aligned}
\mu_{k} \partial_{x x} u_{k} & =\mu_{k+1} \partial_{x x} u_{k+1}, \\
v_{k} \partial_{x x x} u_{k} & =v_{k+1} \partial_{x x x} u_{k+1}
\end{aligned}
$$

hold true.

Corollary 14. The solution of problems (67)-(70) has the following form:

$$
\begin{aligned}
u & =\left(\begin{array}{ll}
1 & 0
\end{array}\right)\left(\begin{array}{l}
u \\
v
\end{array}\right)=\left(\begin{array}{ll}
1 & 0
\end{array}\right) F_{n+}^{-1}\left[e^{-\lambda^{2} t} \tilde{f}(\lambda)\right] \\
& =\left(\begin{array}{ll}
1 & 0
\end{array}\right) \int_{0}^{\infty} v_{k}^{*}(\xi, \lambda)\left(\begin{array}{l}
1 \\
1
\end{array}\right) f_{k}(\xi) d \xi .
\end{aligned}
$$

\section{Stress Produced in the Elastic Semi-Infinite Solid by Pressure}

Let us consider a problem about distribution of tension in an $n+1$-layer elastic semi-infinite solid:

$$
I_{n}^{+} \times R=\left\{(x, y): x \in I_{n}^{+}, y \in R\right\} .
$$


In the case of plane, the strain vector of displacement $\bar{u}_{i}$ has components $u_{i}, v_{i}, 0$. Introduce Airy stress function [10]

$$
p=\sum_{k=1}^{n+1} \theta\left(x-l_{k-1}\right) \theta\left(l_{k}-x\right) p_{k}(y, x)
$$

as a solution to system of differential equations

$$
\left(\partial_{y y}+\partial_{x x}\right)^{2} p_{k}=0, \quad x \in\left(l_{k-1}, l_{k}\right), \quad k=1, \ldots, n+1
$$

with boundary conditions

$$
\begin{aligned}
& \sigma_{1 x}=-p^{\prime \prime}(y), \\
& \tau_{1 x}=0,
\end{aligned}
$$

$$
\text { if } x=0
$$

and internal boundary conditions at the points $x=l_{k}$

$$
\begin{aligned}
\sigma_{k x} & =\sigma_{k+1 x}, \\
\tau_{k x y} & =\tau_{k+1 x y}, \\
u_{k} & =u_{k+1}, \\
v_{k} & =v_{k+1},
\end{aligned}
$$

where $\sigma_{k x}$ is the normal stress and $t_{k x y}$ is the shearing stresses. Fourier transform of Airy stress function with respect to $y$

$$
\bar{p}=\sum_{k=1}^{n+1} \theta\left(x-l_{k-1}\right) \theta\left(l_{k}-x\right) \bar{p}_{k}(y, x)
$$

is the solution to system of differential equations

$$
\begin{array}{r}
\left(-\xi^{2}+d_{x x}\right)^{2} \bar{p}_{k}=0, \\
x \in\left(l_{k-1}, l_{k}\right), k=1, \ldots, n+1
\end{array}
$$

with boundary conditions

$$
\begin{aligned}
\bar{\sigma}_{1 x} & =\xi^{2} \bar{p}(\xi), \\
\bar{\tau}_{1 x} & =0,
\end{aligned}
$$

$$
\text { if } x=0
$$

and internal boundary conditions at the points $x=l_{k}$

$$
\begin{aligned}
\bar{\sigma}_{k x} & =\bar{\sigma}_{k+1 x}, \\
\bar{\tau}_{k x y} & =\bar{\tau}_{k+1 x y}, \\
\bar{u}_{k} & =\bar{u}_{k+1}, \\
\bar{v}_{k} & =\bar{v}_{k+1} .
\end{aligned}
$$

At the beginning, we will solve an auxiliary vector mixed boundary value problem. Let

$$
\left(\begin{array}{l}
\bar{p} \\
\bar{q}
\end{array}\right)=\sum_{k=1}^{n+1} \theta\left(x-l_{k-1}\right) \theta\left(l_{k}-x\right)\left(\begin{array}{l}
\bar{p}_{k} \\
\bar{q}_{k}
\end{array}\right)
$$

be a solution to the system of differential equations

$$
-\xi^{2}\left(\begin{array}{l}
\bar{p}_{k} \\
\bar{q}_{k}
\end{array}\right)+\left(\begin{array}{cc}
1 & -1 \\
0 & 1
\end{array}\right) d_{x x}\left(\begin{array}{l}
\bar{p}_{k} \\
\bar{q}_{k}
\end{array}\right)=0,
$$

with boundary conditions

$$
\begin{aligned}
\bar{p}_{1} & =-\bar{p}(\xi), \\
\partial_{x} \bar{p}_{1} & =0
\end{aligned}
$$

and internal boundary conditions at the points $x=l_{k}$

$$
\begin{aligned}
& \left(\begin{array}{cc}
1 & 0 \\
\frac{1+\sigma_{k}}{E_{k}} & -\frac{1-\sigma_{k}^{2}}{E_{k}}
\end{array}\right)\left(\begin{array}{l}
\bar{p}_{k} \\
\bar{q}_{k}
\end{array}\right) \\
& =\left(\begin{array}{cc}
1 & 0 \\
\frac{1+\sigma_{k+1}}{E_{k+1}} & -\frac{1-\sigma_{k+1}^{2}}{E_{k+1}}
\end{array}\right)\left(\begin{array}{c}
\bar{p}_{k+1} \\
\bar{q}_{k+1}
\end{array}\right), \\
& \left(\begin{array}{cc}
1 & 0 \\
\frac{1+\sigma_{k}}{E_{k}} & \frac{1-\sigma_{k}^{2}}{E_{k}}
\end{array}\right) \partial_{x}\left(\begin{array}{l}
\bar{p}_{k} \\
\bar{q}_{k}
\end{array}\right) \\
& =\left(\begin{array}{cc}
1 & 0 \\
\frac{1+\sigma_{k+1}}{E_{k+1}} & \frac{1-\sigma_{k+1}^{2}}{E_{k+1}}
\end{array}\right) \partial_{x}\left(\begin{array}{l}
\bar{p}_{k+1} \\
\bar{q}_{k+1}
\end{array}\right) .
\end{aligned}
$$

Lemma 15. The solution of problems (101)-(104) in the Fourier images takes the form

$$
\left(\begin{array}{l}
\bar{p} \\
\bar{q}
\end{array}\right)=-F_{n+}^{-1}\left[\frac{1}{\xi^{2}+\lambda^{2}}\right]\left(\begin{array}{c}
\bar{p}(\xi) \\
0
\end{array}\right)
$$

and in the Fourier originals has the form

$$
\left(\begin{array}{l}
p \\
q
\end{array}\right)=-\frac{1}{2 \pi} \int_{0}^{\infty} F_{n+}^{-1}\left[e^{-\lambda|y-\eta|}\right]\left(\begin{array}{c}
p(\eta) \\
0
\end{array}\right) d \eta
$$

Inverse Fourier transform $F_{n+}^{-1}$ is constructed in accordance with (44).

Theorem 16. Let $(\bar{p})$ be a solution of vector problems (101)(104); then,

$$
\bar{p}_{k}=\left(\begin{array}{ll}
1 & 0
\end{array}\right)\left(\begin{array}{c}
\bar{p}_{k} \\
\bar{q}_{k}
\end{array}\right), \quad k=1, \ldots, n+1
$$

is the solution of scalar problems (75)-(76).

Proof. In accordance with (101), the function $\mathrm{p}$ is a solution of iterated Laplace equation (93):

$$
\left(-\xi^{2}+d_{x x}\right)^{2} p_{k}=d_{x x}\left(-\xi^{2}+d_{x x}\right) q_{k}=0 .
$$


Calculating the components $\bar{u}_{k}$ and $\bar{v}_{k}$ of strain vector of displacement on the basis of [10], we get

$$
\begin{aligned}
\bar{v}_{k} & =\frac{1+\sigma_{k}}{2 \pi E}\left(\left(1-\sigma_{k}\right) \bar{p}_{k}^{\prime \prime}+\sigma_{k} \xi^{2} \bar{p}_{k}\right) \\
& =\frac{1+\sigma_{k}}{2 \pi E}\left(\left(1-\sigma_{k}\right)\left(\xi^{2} \bar{p}_{k}-\xi^{2} \bar{q}_{k}\right)+\sigma_{k} \xi^{2} \bar{p}_{k}\right) \\
& =\frac{1+\sigma_{k}}{2 \pi E}\left(\xi^{2} \bar{p}_{k}-\left(1-\xi^{2}\right) \bar{q}_{k}\right), \\
\bar{u}_{k} & =\frac{1+\sigma_{k}}{2 \pi E}\left(\left(1-\sigma_{k}\right) \bar{p}_{k}^{\prime \prime \prime}-\left(2-\sigma_{k}\right) \xi^{2} \bar{p}_{k}^{\prime}\right) \\
& =\frac{1+\sigma_{k}}{2 \pi E}\left(\left(1-\sigma_{k}\right)\left(\xi^{2} \bar{p}_{k}^{\prime}-\xi^{2} \bar{q}_{k}^{\prime}\right)-\left(2-\sigma_{k}\right) \xi^{2} \bar{p}_{k}^{\prime}\right) \\
& =\frac{1+\sigma_{k}}{2 \pi E}\left(-\xi^{2} \bar{p}_{k}-\left(1-\xi^{2}\right) \bar{q}_{k}\right) .
\end{aligned}
$$
hold.

As a result, the internal boundary conditions (103)-(104)

Corollary 17. The solution of problems (93)-(95) has the form

$$
\begin{aligned}
p & =\left(\begin{array}{ll}
1 & 0
\end{array}\right)\left(\begin{array}{l}
p \\
q
\end{array}\right) \\
& =-\left(\begin{array}{ll}
1 & 0
\end{array}\right) \frac{1}{2 \pi} \int_{0}^{\infty} F_{n+}^{-1}\left[e^{-\lambda|y-\eta|}\right]\left(\begin{array}{c}
p(\eta) \\
0
\end{array}\right) d \eta .
\end{aligned}
$$

\section{Conclusion}

Usage of the integral Fourier matrix transforms with piecewise trigonometric kernels method allows us to solve internal boundary conditions problems. Internal boundary conditions problems arise in mathematical modeling of heat conduction and stress produced in the piecewise homogeneous media.

\section{Competing Interests}

The authors declare that they have no competing interests.

\section{References}

[1] R. Bracewell, The Fourier Transform and Its Applications, McGraw-Hill College, 3rd edition, 1999.

[2] J. F. James, A Student's Guide to Fourier Transforms, Cambridge University Press, Cambridge, UK, 1995.

[3] J. T. W. Keorner, Fourier Analysis, Cambridge University Press, Cambridge, UK, 1988.

[4] L. Grafakos, Classical and Modern Fourier Analysis, PrenticeHall, Upper Saddle River, NJ, USA, 2004.

[5] B. Osgood, The Fourier Transform and Its Applications, Stanford University, 2009.

[6] M. A. Pinsky, Introduction to Fourier Analysis and Wavelets, Brooks/Cole, 2002.

[7] M. A. Pinsky, "Fourier inversion for piecewise smooth functions in several variables," Proceedings of the American Mathematical Society, vol. 118, no. 3, pp. 903-910, 1993.
[8] A. D. Polyanin and A. V. Manzhirov, Handbook of Integral Equations, CRC Press, Boca Raton, Fla, USA, 1998.

[9] M. S. Salih, Fourier Transform Applications, InTech, Rijeka, Croatia, 2012.

[10] I. N. Sneddon, Fourier Transforms, McGraw-Hill, 1951.

[11] E. Stein and R. Shakarchi, Fourier Analysis: An Introduction, Princeton University Press, Princeton, NJ, USA, 2003.

[12] E. C. Titchmarsh, Introduction to the Theory of Fourier Integrals, Clarendon, Oxford, UK, 1948.

[13] G.-C. Kuo, Y.-H. Hu, W.-L. Liaw, K.-J. Wang, and K.-Y. Kung, "Transient temperature solutions of a cylindrical fin," WSEAS Transactions on Mathematics, vol. 10, no. 2, pp. 47-55, 2011.

[14] K.-T. Chiang, G. C. Kuo, K.-J. Wang, Y. F. Hsiao, and K.-Y. Kung, "Transient temperature analysis of a cylindrical heat equation," WSEAS Transactions on Mathematics, vol. 7, no. 8, pp. 309-319, 2009.

[15] P.-Y. Wang, G.-C. Kuo, Y.-H. Hu, and W.-L. Liaw, "Transient temperature solutions of a cylindrical fin with lateral heat loss," WSEAS Transactions on Mathematics, vol. 11, no. 10, pp. 918952, 2012.

[16] J. S. Ufljand, Integral'nye preobrazovanija $v$ zadachah teorii uprugosti, Nauka, Leningrad, Russia, 1967.

[17] L. S. Najda, "Gibridnye integral'nye preobrazovanija tipa Hankelja-Lezhandra," Matematicheskie Metody Analiza Dinamicheskih Sistem, Harkov, vol. 8, pp. 132-135, 1984.

[18] V. S. Procenko and A. I. Solov'jov, “Gibridnye integral'nye preobrazovanija i ih prilozhenija v teorii uprugosti neodnorodnyh sred," Prikladnaja Mehanika, vol. 1, no. 13, pp. 62-67, 1982.

[19] M. P. Lenjuk, "Integral'noe preobrazovanie Fur'e na kusochnoodnorodnoj poluprjamoj," Izvestiya Vysshikh Uchebnykh Zavedenii. Matematika, vol. 4, pp. 14-18, 1989.

[20] F. R. Gantmacher, The Theory of Matrices, Reprinted by American Mathematical Society, AMS Chelsea, 2000.

[21] O. Yaremko, V. Selutin, and N. Yaremko, "The fourier transform with piecewise trigonometric kernels and its applications," WSEAS Transactions on Mathematics, vol. 13, pp. 615-625, 2014.

[22] F. M. Mors and G. Fishbah, Methods of Theoretical Physics, part 1, McGraw-Hill, New York, NY, USA, 1953. 


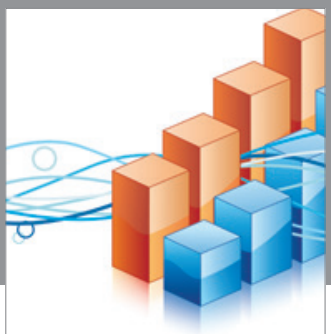

Advances in

Operations Research

vatem alat4

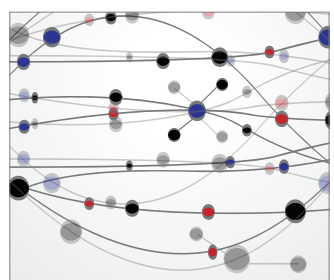

\section{The Scientific} World Journal
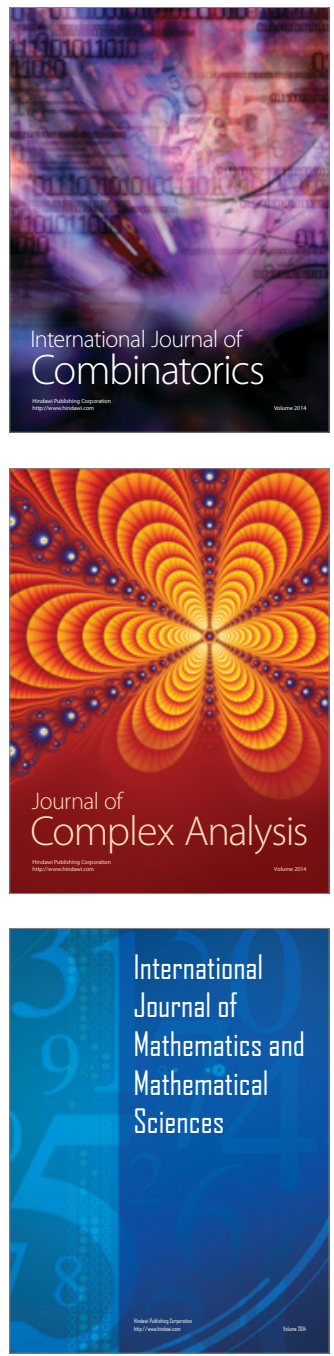
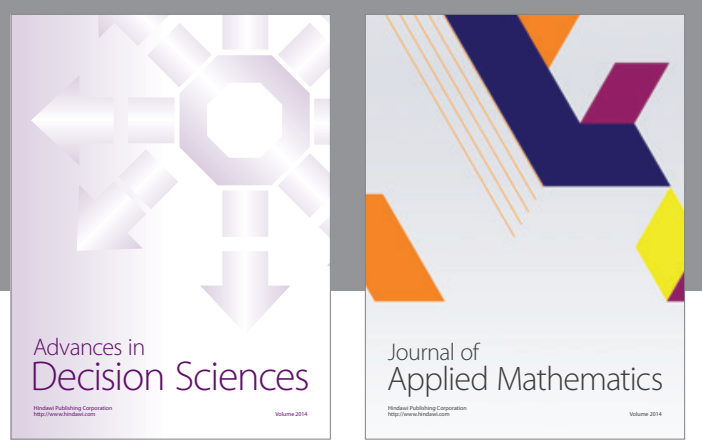

Algebra

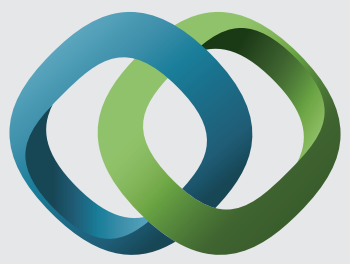

\section{Hindawi}

Submit your manuscripts at

http://www.hindawi.com
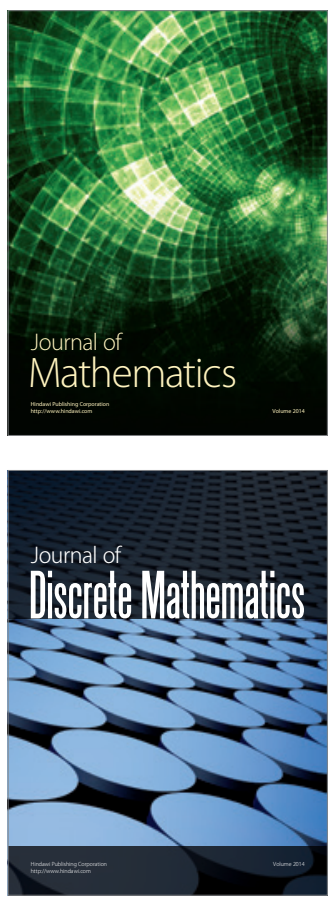

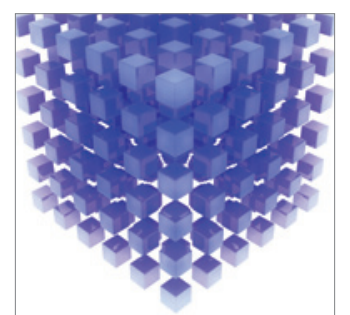

Mathematical Problems in Engineering
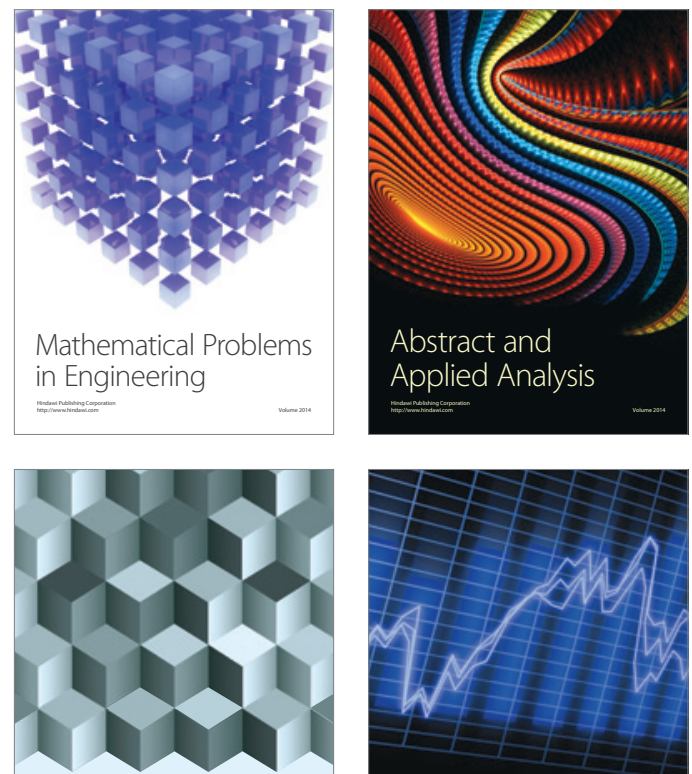

Journal of

Function Spaces

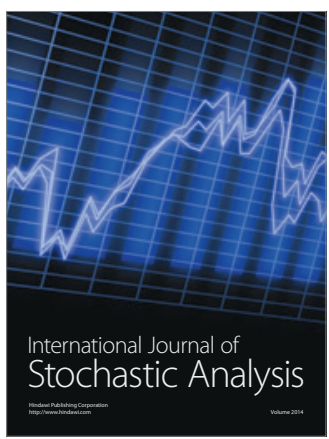

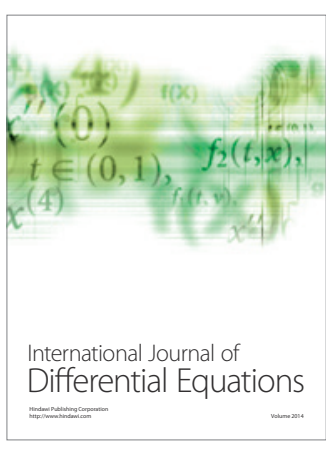
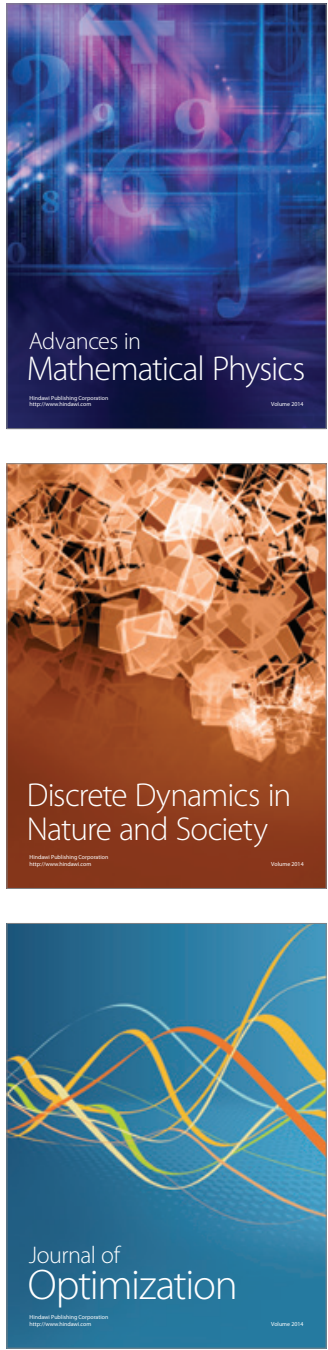